\title{
Fourier analysis of 2-point Hermite interpolatory subdivision schemes
}

\author{
Serge Dubuc, Daniel Lemire`and Jean-Louis Merrien ${ }^{\ddagger}$
}

$12 / 14 / 2000$

\begin{abstract}
Two subdivision schemes with Hermite data on $\mathbb{Z}$ are studied. These schemes use 2 or 7 parameters respectively depending on whether Hermite data involve only first derivatives or include second derivatives. For a large region in the parameter space, the schemes are convergent in the space of Schwartz distributions. The Fourier transform of any interpolating function can be computed through products of matrices of order 2 or 3 . The Fourier transform is related to a specific system of functional equations whose analytic solution is unique except for a multiplicative constant. The main arguments for these results come from Paley-Wiener-Schwartz theorem on the characterization of the Fourier transforms of distributions with compact support and a theorem of Artzrouni about convergent products of matrices.
\end{abstract}

Math Subject Classification: 40A20, 42A38, 46F12, 65D05, 65D10

Keywords and Phrases: Hermite interpolation, curve fitting, subdivision, Fourier transform, distributions, convergence of infinite products, products of matrices.

\section{Introduction}

Hermite interpolatory subdivision schemes have been introduced by Merrien [10, 11] and Dyn-Levin $[5,6]$. These authors studied the $C^{k}$-convergence of these schemes. What we would like to do here is to compute the Fourier transform of these interpolants and to provide an additional tool for studying these schemes and for generating functions which are not necessarily of class $C^{1}$ or $C^{2}$.

${ }^{*}$ S. Dubuc, Département de mathématiques et de statistique, Université de Montréal, C.P. 6128 Succursale Centre-ville, Montréal (Québec), H3C 3J7 Canada, email: dubucs@dms.umontreal.ca

${ }^{\dagger}$ D. Lemire, Ondelette Inc., 2059, rue Wurtele, Montréal (Québec), H2K 2P8 Canada, email: lemire@ondelette.com

${ }^{\ddagger}$ J.-L. Merrien, INSA de Rennes, 20 av. des Buttes de Coësmes, CS 14315, 35043 RENNES CEDEX, France, email: Jean-Louis.Merrien@insa-rennes.fr 
As we will see, it is possible to define the notion of convergence of Hermite interpolatory subdivision schemes in the space of Schwartz distributions. The study of the convergence of these schemes on the space $\mathcal{D}(\mathbb{R})^{\prime}$ can be done through the Fourier transform provided that sufficiently general results about convergence of infinite products of matrices are available. We came to this approach after previous works of Deslauriers-Dubuc [3] and of Deslauriers-Dubois-Dubuc [4]. These authors considered interpolatory subdivision schemes which were not of Hermite type however. In this situation, the Fourier transform allowed the study of the convergence of some schemes with the help of products of trigonometrical polynomials (and not of matrices).

We would like to point out two previous works that use harmonic analysis and which have points in common with our article. A first analysis has been written by Hervé [7] about wavelets in two or more dimensions. He did not study convergence conditions, but he was involved in products of matrices. Another analysis, in subdivision schemes this time, is due to Kobbelt [8]. He proved a general convergence criterion for arbitrary interpolatory schemes via discrete Fourier transform (instead of the Fourier integral transform).

We now summarize the contents of the paper. In Section 2, we study a first Hermite interpolation scheme on $\mathbb{R}$ of a function and its first derivative with data on $\mathbb{Z}$. This scheme called $H S 21$ depends on two parameters $\alpha$ and $\beta$. We first recall the conditions which insure $C^{1}$-convergence on $\mathbb{R}$. Then we evaluate the Fourier transform of the interpolating functions and we give the first properties. The Paley-WienerSchwartz theorem on the characterization of the Fourier transform of a distribution with compact support and a convergence theorem of infinite products of matrices proposed by Artzrouni allow us to conclude to the convergence of the scheme in the space of distributions $\mathcal{D}(\mathbb{R})^{\prime}$ for a large region in the parameter space: $\beta \in(-3,1)$ with no restriction on $\alpha$. Moreover, the Fourier transforms of two basic distributions in the Hermite subdivision scheme is the unique (except for a multiplicative constant) analytic solution of a system of two functional equations.

Section 3 is devoted to the study of a second scheme where we interpolate not only a function and its first derivative but also its second derivative. This scheme is called HS22 and depends on 7 parameters: $\beta_{1}$ and $\alpha_{i}, \beta_{i}, \gamma_{i}, i=2,3$. We prove analogous properties to the previous scheme depending on the parameters introduced in the algorithm. In particular, we get a satisfactory convergence in distribution of the scheme when $\beta_{2}$ and $\gamma_{3} \in(-3 / 2,1 / 2), \gamma_{3} \in(-3 / 2,1 / 2), \beta_{1}+2 \beta_{2}=\gamma_{2}+2 \gamma_{3}=1$ and no restriction on $\alpha_{2}, \alpha_{3}$. Most proofs are similar to the previous ones. However, a few matrix tools must be improved.

\section{The Hermite subdivision scheme $H S 21$}

We recall Merrien's construction [10]. We suppose that the function $f$ and its first derivative $p$ are known on $\mathbb{Z}$. Precisely, we have two sequences $\left\{y_{k}, y_{k}^{\prime}\right\}_{k \in \mathbb{Z}}$ and we 
suppose that $f(k)=y_{k}, p(k)=y_{k}^{\prime}$. We build $f$ and $p$ on $\mathbb{Z} / 2^{n}$ by induction. At step $n$, set $h=1 / 2^{n}$ and $D_{n}=\{x=j h, j \in \mathbb{Z}\}$. If $a=j h$ and $b=(j+1) h$ are two consecutive points of $D_{n}$, we compute $f$ and $p$ at $x=(a+b) / 2$ which is the midpoint of $[a, b]$ by the formulae:

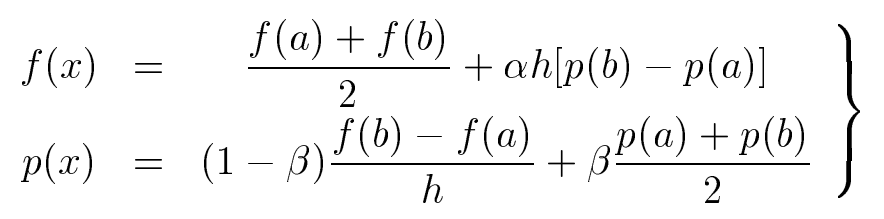

Hence $f$ and $p$ are defined on $D_{n+1}$. The construction depends on two parameters $\alpha$ and $\beta$. When we iterate the process, we define $f$ and $p$ on the set of dyadic numbers $D_{\infty}=\bigcup D_{n}$ which is dense in $\mathbb{R}$. For some values of the parameters the functions $f$ and $p$ may be uniformly continuous on $D_{\infty}$ so that they may be extended to $\mathbb{R}$; sometimes we have in addition: $p=f^{\prime}$. In these cases the algorithm is said to be $C^{1}$ convergent.

$$
\text { If we define } U_{n}^{i}=\left(\begin{array}{c}
p\left((i+1) 2^{-n}\right)-p\left(i 2^{-n}\right) \\
2^{n}\left(f\left((i+1) 2^{-n}\right)-f\left(i 2^{-n}\right)\right)-\left(p\left((i+1) 2^{-n}\right)+p\left(i 2^{-n}\right)\right) / 2
\end{array}\right),
$$

it is easy to prove that: $U_{n+1}^{2 i+1}=\Lambda_{1} U_{n}^{i}$ and $U_{n+1}^{2 i}=\Lambda_{-1} U_{n}^{i}$ where

$\Lambda_{\varepsilon}=\left(\begin{array}{cc}\frac{1}{2} & \varepsilon(1-\beta) \\ \varepsilon \frac{8 \alpha+1}{4} & \frac{1+\beta}{2}\end{array}\right), \varepsilon= \pm 1$. Then the scheme is $C^{1}$-convergent if and only if the generalized spectral radius of $\left\{\Lambda_{1}, \Lambda_{-1}\right\}$ is $<1$. An equivalent condition is that there exists a matrix norm $\|\cdot\|$ such that: $\left\|\Lambda_{\varepsilon}\right\|<1, \varepsilon= \pm 1$. This result can be proved by techniques which are described in [11]. Details on generalized spectral radii can be found in Daubechies-Lagarias [2], but one knows that their evaluation is difficult or even impossible.

Remark 1: If $\alpha=-1 / 8, \beta=-1 / 2$, then $f$ is the Hermite interpolating cubic polynomial between two consecutive integers. When $\alpha=-1 / 8, \beta=-1$, then $f$ is the quadratic interpolating spline with knots on $\mathbb{Z} / 2$.

\subsection{Elementary properties of $H S 21$}

We describe some elementary properties of Hermite subdivision schemes. We will use those properties later. The first one is the linearity of the scheme.

Lemma 1 Let $\left\{y_{k}, y_{k}^{\prime}, \tilde{y}_{k}, \tilde{y}_{k}^{\prime}\right\}_{k \in \mathbb{Z}}$ be 4 sequences. Assume that both pairs $(f, p)$ and $(\tilde{f}, \tilde{p})$ are built by the subdivision scheme from: $f(k)=y_{k}, p(k)=y_{k}^{\prime}, \tilde{f}(k)=\tilde{y}_{k}$, $\tilde{p}(k)=\tilde{y}_{k}^{\prime}$, then the pair of functions $(f+\tilde{f}, p+\tilde{p})$ are obtained by the subdivision scheme from the sequences: $\left\{y_{k}+\tilde{y}_{k}, y_{k}^{\prime}+\tilde{y}_{k}^{\prime}\right\}_{k \in \mathbb{Z}}$.

Similarly, if $c \in \mathbb{R}$, the pair of functions $(c f, c p)$ is obtained by the scheme from the sequences: $\left\{c y_{k}, c y_{k}^{\prime}\right\}_{k \in \mathbb{Z}}$.

Then we have a second lemma about translation and scale change. 
Lemma 2 Let $\left\{y_{k}, y_{k}^{\prime}\right\}_{k \in \mathbb{Z}}$ be two sequences from which we build the pair of functions $(f, p)$ by the subdivision scheme with $f(k)=y_{k}, p(k)=y_{k}^{\prime}$. If $c \in \mathbb{Z}$, then the pair of functions $(f(x+c), p(x+c))$ is obtained by the scheme from the sequences: $\left\{y_{k+c}, y_{k+c}^{\prime}\right\}_{k \in \mathbb{Z}}$.

The pair of functions $(f(x / 2), p(x / 2) / 2)$ is built from the sequences: $\{f(k / 2), p(k / 2) / 2\}_{k \in \mathbb{Z}}$.

There are two basic solutions of our recursive system (1): the first one is the pair $\left(f_{0}, p_{0}\right)$ which is solution of $(1)$ with data $f_{0}(k)=\delta_{k, 0}, p_{0}(k)=0, k \in \mathbb{Z}$ and the second one is the pair $\left(f_{1}, p_{1}\right)$ which is solution of $(1)$ with data $f_{1}(k)=0, p_{1}(k)=\delta_{k, 0}, k \in \mathbb{Z}$. These two pairs are important because with linear combinations of their tranlates we can get all solutions $(f, p)$ of (1). For any dyadic number $x$ :

$$
\left.\begin{array}{l}
f(x)=\sum_{k=-\infty}^{\infty}\left[f(k) f_{0}(x-k)+p(k) f_{1}(x-k)\right] \\
p(x)=\sum_{k=-\infty}^{\infty}\left[f(k) p_{0}(x-k)+p(k) p_{1}(x-k)\right]
\end{array}\right\}
$$

Notice that both sums are finite as the supports of $f_{i}, p_{i}, i=0,1$ lie in the set $[-1,1]$.

Now, using relation (1) which is applied to the pair of functions $\left(f(x)=f_{0}(x / 2), p(x)=p_{0}(x / 2) / 2\right)$ and then to the pair $\left(f_{1}(x / 2), p_{1}(x / 2) / 2\right)$, after evaluations of the functions $f_{0}, p_{0}, f_{1}, p_{1}$ at the half-integers, we obtain a system of functional equations for $f_{0}, p_{0}, f_{1}, p_{1}$.

$$
\left.\begin{array}{rl}
f_{0}(x / 2) & =\frac{1}{2} f_{0}(x-1)+f_{0}(x)+\frac{1}{2} f_{0}(x+1)-\frac{1-\beta}{2} f_{1}(x-1)+\frac{1-\beta}{2} f_{1}(x+1), \\
p_{0}(x / 2) / 2 & =\frac{1}{2} p_{0}(x-1)+p_{0}(x)+\frac{1}{2} p_{0}(x+1)-\frac{1-\beta}{2} p_{1}(x-1)+\frac{1-\beta}{2} p_{1}(x+1), \\
f_{1}(x / 2) & =-\alpha f_{0}(x-1)+\alpha f_{0}(x+1)+\frac{\beta}{4} f_{1}(x-1)+\frac{1}{2} f_{1}(x)+\frac{\beta}{4} f_{1}(x+1), \\
p_{1}(x / 2) / 2 & =-\alpha p_{0}(x-1)+\alpha p_{0}(x+1)+\frac{\beta}{4} p_{1}(x-1)+\frac{1}{2} p_{1}(x)+\frac{\beta}{4} p_{1}(x+1) .
\end{array}\right\}
$$

\section{$2.2 \quad$ Fourier transform of $H S 21$}

In this section, we suppose that the system (3) of functional equations is valid not only whenever $x$ is a dyadic number, but also whenever $x$ is an arbitrary real number. Moreover we assume that $f_{0}, p_{0}, f_{1}, p_{1} \in L^{1}(\mathbb{R})$. Now, we compute the Fourier transform $\hat{f}$ of a function $f$ by

$$
\hat{f}(\xi)=\int_{-\infty}^{\infty} f(x) e^{-i \xi x} d x
$$

Using this Fourier operator on each equation of the system (3), we get:

$$
\left(\begin{array}{c}
\hat{f}_{0}(\xi) \\
\hat{f}_{1}(\xi)
\end{array}\right)=A(\xi / 2)\left(\begin{array}{c}
\hat{f}_{0}(\xi / 2) \\
\hat{f}_{1}(\xi / 2)
\end{array}\right),\left(\begin{array}{c}
\hat{p}_{0}(\xi) \\
\hat{p}_{1}(\xi)
\end{array}\right)=2 A(\xi / 2)\left(\begin{array}{c}
\hat{p}_{0}(\xi / 2) \\
\hat{p}_{1}(\xi / 2)
\end{array}\right)
$$


where

$$
A(\xi)=\left(\begin{array}{cc}
\frac{1}{2}+\frac{1}{2} \cos \xi & i \frac{1-\beta}{2} \sin \xi \\
i \alpha \sin \xi & \frac{1}{4}+\frac{\beta}{4} \cos \xi
\end{array}\right)
$$

We have two vector equations in (4). To study them, we now look at the product of matrices:

$$
P_{n}(\xi)=A(\xi / 2) A(\xi / 4) \ldots A\left(\xi / 2^{n}\right) .
$$

Precisely, we look for conditions on the parameters $\alpha, \beta$ to get convergence of the sequence of matrices $P_{n}(\xi)$. This convergence should happen for every real or complex value of $\xi$. The study of this sequence for complex values of $\xi$ is motivated by a generalization of Paley-Wiener theorem as proposed by Schwartz [12].

Theorem 3 (Schwartz) Let $F$ be a continuous function on the real axis which is the Fourier transform of a tempered distribution $T$. The support of $T$ is contained in $[-C, C]$ if and only if $F$ may be extended on the complex plane to an entire function of exponential type $\leq C$.

We recall that an entire function $F(z)$ is of exponential type $\leq C$ if

$$
\limsup _{|z| \rightarrow \infty} \frac{\log |F(z)|}{|z|} \leq C
$$

To study the convergence of the matrix products $P_{n}(\xi)$, we will need a lemma to bound the moduli of the elements of $P_{n}(\xi)$. We will also need a convergence criterion which has been found by Artzrouni [1].

Lemma 4 Let $\|\cdot\|$ be a matrix norm on the space of complex matrices of order $d$, $\mathbb{C}^{d \times d}$, and let $A_{n}$ be a sequence in this space such that $\left\|A_{n}\right\| \leq 1+\varepsilon_{n}$, with $\varepsilon_{n} \geq 0$. Set $P_{0}=I, P_{n}=P_{n-1} A_{n}, n=1,2, \ldots$ Then $\left\|P_{n}\right\| \leq e^{\sum_{k=1}^{n} \overline{\varepsilon_{k}}}$ and the modulus of each component of $P_{n}$ is bounded by $C e^{\sum_{k=1}^{n} \varepsilon_{k}}$ where $C$ is a constant which depends only on the matrix norm $\|\cdot\|$.

Proof: Since we notice that $1+\varepsilon_{n} \leq e^{\varepsilon_{n}}$, we get the first result by induction.

Then, for $A=\left(a_{i j}\right) \in \mathbb{C}^{d \times d}$, we choose a new vector norm $N(A)=\max \left\{\left|a_{i j}\right|\right\}$. We know that there exists a constant $C_{N}$ such that for all $A \in \mathbb{C}^{d \times d}, N(A) \leq C_{N}\|A\|$. Then we get the second upper bound.

Theorem 5 (Artzrouni) Let $\|\cdot\|$ be a matrix norm on $\mathbb{C}^{d \times d}$. Let $M_{n}$ be a sequence in $\mathbb{C}^{d \times d}$ such that for all $n \in \mathbb{N},\left\|M_{n}\right\|=1$ and for all $m \in \mathbb{N}$ the sequence of matrices $M_{m} M_{m+1} \ldots M_{n}, n \geq m$ converges. If $N_{n}$ is a sequence in $\mathbb{C}^{d \times d}$ such that $\sum_{n=1}^{\infty}\left\|N_{n}-M_{n}\right\|<\infty$, then the sequence of matrices $N_{1} N_{2} \ldots N_{n}$ converges.

We are now ready for the main result. 
Theorem 6 If $-5<\beta \leq 3$, then for all complex numbers $\xi$ the sequence of matrices $P_{n}(\xi)$ defined in (5) converges and the convergence is uniform on any bounded domain. As functions of $\xi$, the four components of the limit matrix $P(\xi)$ are entire functions of exponential type $\leq 1$.

Proof: Let us start by proving that the moduli of all components of $P_{n}(z)$ are uniformly bounded whenever $z \in \Omega$, a bounded domain in $\mathbb{C}$.

If $A=\left(a_{i j}\right)$ is a matrix in $\mathbb{C}^{2 \times 2}$, we recall that $\|A\|_{\infty}=\max _{i}\left\{\left|a_{i 1}\right|+\left|a_{i 2}\right|\right\}$. Then, we use Lemma 4 with the matrices $A_{n}=A\left(z / 2^{n}\right)$ and the sequence

$$
\varepsilon_{n}=\max \left[0, \sup _{|z| \in \Omega}\left(\left\|A\left(z / 2^{n}\right)\right\|_{\infty}-1\right)\right] .
$$

If the components of the matrix $A(z)$ are $a_{i j}(z)$, these functions are analytic at $z=0$ with $A(0)=\left(\begin{array}{cc}1 & 0 \\ 0 & \frac{1+\beta}{4}\end{array}\right)$ therefore $\varepsilon_{n}=O\left(1 / 2^{n}\right)$ (remembering the hypothesis $-5<$ $\beta \leq 3)$. Lemma 4 shows that the moduli of all components of the matrices $P_{n}(z)$ are uniformly bounded whenever $z \in \Omega$.

Now, we prove that for all $z \in \mathbb{C}$, the sequence of matrices $P_{n}(z)$ converges. Let $M_{n}$ be the sequence of constant matrices $M=M_{n}=A(0)$ and define the sequence $N_{n}=A\left(z / 2^{n}\right)$. We know that $\left\|N_{n}-M_{n}\right\|_{\infty}=O\left(1 / 2^{n}\right)$. Moreover $\|M\|_{\infty}=1$ and with the hypothesis on $\beta$, the sequence $M^{k}, k \geq 0$ converges. All the hypotheses of Theorem 5 are satisfied, therefore the sequence $P_{n}(z)$ converges.

As the sequence $P_{n}(z)$ converges to a matrix $P(z)$ and as the moduli of the components of the matrices $P_{n}(z)$ are uniformly bounded whenever $z \in \Omega$, the Lebesgue dominated convergence theorem and the Cauchy formula give us the proof that all the components of the matrix $P(z)$ are analytic in $z$ and that the convergence of the sequence $P_{n}(z)$ to $P(z)$ is uniform on $\Omega$.

Finally, let us verify that each element of the matrix $P(z)$ is an entire function of exponential type. Firstly, there exists a real positive number $C$ which depends on the parameters $\alpha, \beta$ such that for all $z \in \mathbb{C},\|A(z)\|_{\infty} \leq C e^{|z|}$. Secondly, we know that there exists a real positive number $M$ (depending on the parameters again) such that for all $z \in \mathbb{C},|z| \leq 1,\|P(z)\|_{\infty} \leq M$.

Let $z$ be a complex number such that $2^{n} \leq|z| \leq 2^{n+1}$. As $P(z)=A(z / 2) A(z / 4) \ldots A\left(z / 2^{n}\right) P\left(z / 2^{n+1}\right)$, we obtain the bound

$$
\|P(z)\|_{\infty} \leq \| P\left(z / 2^{n+1}\left\|_{\infty} \prod_{k=1}^{n}\right\| A\left(z / 2^{k}\right) \|_{\infty} \leq M \prod_{k=1}^{n}\left[C e^{z / 2^{k}}\right] \leq M C^{n} e^{|z|}\right.
$$

Hence $\limsup \operatorname{su}_{|z| \rightarrow \infty} \frac{\log || P(z) \|_{\infty}}{|z|} \leq 1$ and the functions composing the matrix $P(z)$ are entire functions of exponential type $\leq 1$.

The Paley-Wiener theorem implies the following corollary:

Corollary 7 Let us assume that $-5<\beta \leq 3$. Then each function composing the limit matrix $P(z)=\lim P_{n}(z)$ is the Fourier transform of a distribution whose support lies in the interval $[-1,1]$. 


\subsection{Schwartz distributions associated with the scheme}

We will link the computation of Fourier transforms of the previous subsection with the limit matrix $P(\xi)$. This link will come from four sequences of Schwartz distributions. We set

$$
\begin{aligned}
T_{i}^{(n)} & =\frac{1}{2^{n}} \sum_{m} f_{i}\left(m / 2^{n}\right) \delta_{m / 2^{n}}, i=0,1, \\
U_{i}^{(n)} & =\frac{1}{2^{n}} \sum_{m} p_{i}\left(m / 2^{n}\right) \delta_{m / 2^{n}}, i=0,1,
\end{aligned}
$$

where $\delta_{h}$ is the Dirac distribution at point $h$ defined by $\delta_{h}(\phi)=\phi(h)$.

Notice that these sums are finite and that the distributions are compactly supported, the supports of $f_{i}, p_{i}, i=0,1$ being in $[-1,1]$.

We evaluate the Fourier transform of these four distributions:

$$
\hat{T}_{i}^{(n)}(\xi)=T_{i}^{(n)}\left(e^{-i \xi x}\right), \hat{U}_{i}^{(n)}(\xi)=U_{i}^{(n)}\left(e^{-i \xi x}\right), i=0,1 .
$$

Hence using the equalities (3), we verify that two simple inductions link both Fourier transforms through the matrix $A(\xi)$. Indeed,

$$
\hat{T}_{0}^{(n+1)}(\xi)=\frac{1}{2^{n+1}} \sum_{m} f_{0}\left(m / 2^{n+1}\right) e^{-i \xi m / 2^{n+1}} .
$$

In this last equation, we substitute to $f_{0}\left(m / 2^{n+1}\right)$ by means of the first equation of system (3) with $x=m / 2^{n}$ to obtain a first recursion:

$$
\hat{T}_{0}^{(n+1)}(\xi)=\left[\frac{1}{2}+\frac{1}{2} \cos \left(\frac{\xi}{2}\right)\right] \hat{T}_{0}^{n}(\xi / 2)+i \frac{1-\beta}{2} \sin \left(\frac{\xi}{2}\right) \hat{T}_{1}^{n}(\xi / 2)
$$

Similarly, we can evaluate $\hat{T}_{1}^{(n+1)}(\xi)$ using the third equation of system (3) again at $x=m / 2^{n}$ and we get a second recursion:

$$
\hat{T}_{1}^{(n+1)}(\xi)=i \alpha \sin \left(\frac{\xi}{2}\right) \hat{T}_{0}^{n}(\xi / 2)+\left[\frac{1}{4}+\frac{\beta}{4} \cos \left(\frac{\xi}{2}\right)\right] \hat{T}_{1}^{n}(\xi / 2)
$$

Both recursions may be linked in a single vector recursion through the matrix $A(\xi / 2)$ :

$$
\left(\begin{array}{c}
\hat{T}_{0}^{(n+1)}(\xi) \\
\hat{T}_{1}^{(n+1)}(\xi)
\end{array}\right)=A(\xi / 2)\left(\begin{array}{c}
\hat{T}_{0}^{(n)}(\xi / 2) \\
\hat{T}_{1}^{(n)}(\xi / 2)
\end{array}\right)
$$

Similarly, with the second and fourth equations of system (3), we obtain a second vector recursion:

$$
\left(\begin{array}{c}
\hat{U}_{0}^{(n+1)}(\xi) \\
\hat{U}_{1}^{(n+1)}(\xi)
\end{array}\right)=2 A(\xi / 2)\left(\begin{array}{c}
\hat{U}_{0}^{(n)}(\xi / 2) \\
\hat{U}_{1}^{(n)}(\xi / 2)
\end{array}\right)
$$




$$
\begin{aligned}
& \text { Since }\left(\begin{array}{c}
\hat{T}_{0}^{(0)}(\xi) \\
\hat{T}_{1}^{(0)}(\xi)
\end{array}\right)=\left(\begin{array}{c}
1 \\
0
\end{array}\right) \text { and }\left(\begin{array}{c}
\hat{U}_{0}^{(0)}(\xi) \\
\hat{U}_{1}^{(0)}(\xi)
\end{array}\right)=\left(\begin{array}{c}
0 \\
1
\end{array}\right) \text {, we get } \\
& \left(\begin{array}{c}
\hat{T}_{0}^{(n)}(\xi) \\
\hat{T}_{1}^{(n)}(\xi)
\end{array}\right)=P_{n}(\xi)\left(\begin{array}{l}
1 \\
0
\end{array}\right)
\end{aligned}
$$

and

$$
\left(\begin{array}{c}
\hat{U}_{0}^{(n)}(\xi) \\
\hat{U}_{1}^{(n)}(\xi)
\end{array}\right)=2^{n} P_{n}(\xi)\left(\begin{array}{l}
0 \\
1
\end{array}\right)
$$

The vector sequence $\left(\begin{array}{c}\hat{T}_{0}^{(n)}(\xi) \\ \hat{T}_{1}^{(n)}(\xi)\end{array}\right)$ converges to the first column of the matrix $P(\xi)$.

Theorem 8 If $-5<\beta \leq 3$, then each sequence of distributions $T_{i}^{(n)}$ converges to a distribution $T_{i}, i=0,1$. The vector $\left(\hat{T}_{0}(\xi), \hat{T}_{1}(\xi)\right)^{T}$ is the first column of the matrix $P(\xi)$.

Proof: Let $\phi$ a $C^{\infty}$-function with bounded support, we have the inversion formula $\phi(x)=\frac{1}{2 \pi} \int \hat{\phi}(\xi) e^{-i x \xi} d \xi$. So for $i=0,1$,

$$
\begin{aligned}
T_{i}^{(n)}(\phi) & =\frac{1}{2^{n}} \sum_{m} f_{i}\left(m / 2^{n}\right) \phi\left(m / 2^{n}\right) \\
& =\frac{1}{2 \pi} \int \hat{T}_{i}^{(n)}(-\xi) \hat{\phi}(\xi) d \xi
\end{aligned}
$$

As $n$ tends to $\infty$, we get $\lim _{n \rightarrow \infty} T_{i}^{(n)}(\phi)=\frac{1}{2 \pi} \int \hat{p}_{i+1,1}(-\xi) \hat{\phi}(\xi) d \xi=T_{i}(\phi)$.

$\left(\left(p_{11}(\xi), p_{21}(\xi)\right)^{T}\right.$ is the first column of $P(\xi)$.) Hence $T_{i}^{(n)}$ converges to $T_{i}$. From that, it follows that the Fourier transform $\hat{T}_{i}(\xi)$ is $\lim _{n \rightarrow \infty} \hat{T}_{i}^{(n)}(\xi)=p_{i+1,1}(\xi)$.

We are ready to prove that the subdivision scheme is always convergent in the space of distributions $\mathcal{D}(\mathbb{R})^{\prime}$ whenever $-5<\beta \leq 3$. In the following, we use Schwartz notation for the translation operator $\tau_{h}$ where $h$ is a real number. If $\phi$ is a function in $C_{0}^{\infty}$ and if $T$ is a distribution, then $\tau_{h} \phi(x)=\phi(x-h)$ and $\tau_{h} T(\phi)=T\left(\tau_{-h} \phi\right)$.

Theorem 9 Let us assume that $-5<\beta \leq 3$. If we build the pair $(f, p)$ by the subdivision scheme (1) from the data $\left\{y_{k}, y_{k}^{\prime}\right\}_{k \in \mathbb{Z}}$, then the sequence of distributions $F_{n}=\frac{1}{2^{n}} \sum_{m=-\infty}^{\infty} f\left(m / 2^{n}\right) \delta_{m / 2^{n}}$ converges to the distribution

$$
F=\sum_{k=-\infty}^{\infty}\left[y_{k} \tau_{k} T_{0}+y_{k}^{\prime} \tau_{k} T_{1}\right]
$$


Proof: Let $\phi$ be a function in $C^{\infty}$ with support in $[-N, N]$, then $F_{n}(\phi)=\frac{1}{2^{n}} \sum_{m=-N 2^{n}}^{N 2^{n}} f\left(m / 2^{n}\right) \phi\left(m / 2^{n}\right)$. We use relation (2) to get:

$$
\begin{aligned}
2^{n} F_{n}(\phi) & =\sum_{m=-N 2^{n}}^{N 2^{n}} \sum_{k=-N-1}^{N+1}\left[y_{k} f_{0}\left(m / 2^{n}-k\right)+y_{k}^{\prime} f_{1}\left(m / 2^{n}-k\right)\right] \phi\left(m / 2^{n}\right) \\
& =\sum_{k=-N-1}^{N+1} \sum_{m=-N 2^{n}}^{N 2^{n}}\left[y_{k} f_{0}\left(m / 2^{n}\right)+y_{k}^{\prime} f_{1}\left(m / 2^{n}\right)\right] \phi\left(m / 2^{n}+k\right) \\
& =2^{n} \sum_{k=-N-1}^{N+1}\left[y_{k} T_{0}^{(n)}+y_{k}^{\prime} T_{1}^{(n)}\right]\left(\tau_{k} \phi\right) .
\end{aligned}
$$

As $n$ tends to infinity, the limit of the sequence $F_{n}(\phi)$ is:

$$
\sum_{k=-\infty}^{\infty}\left[y_{k} \tau_{k} T_{0}+y_{k}^{\prime} \tau_{k} T_{1}\right](\phi) .
$$

Theorem 10 If $-3<\beta<1$, then both sequences of distributions $U_{0}^{(n)}, U_{1}^{(n)}$ converge respectively to the distributions $T_{0}^{\prime}, T_{1}^{\prime}$ which are the derivatives of the distributions $T_{0}, T_{1}$.

Proof: Using both relations (5) and (7), we have:

$$
\begin{gathered}
\left(\begin{array}{c}
\hat{U}_{0}^{(n)}(\xi) \\
\hat{U}_{1}^{(n)}(\xi)
\end{array}\right)=2^{n} P_{n-1}(\xi) A\left(\xi / 2^{n}\right)\left(\begin{array}{c}
0 \\
1
\end{array}\right) \\
\left(\begin{array}{c}
\hat{U}_{0}^{(n)}(\xi) \\
\hat{U}_{1}^{(n)}(\xi)
\end{array}\right)=2^{n} P_{n-1}(\xi)\left(\begin{array}{c}
i \frac{1-\beta}{2} \sin \left(\xi / 2^{n}\right) \\
\frac{1}{4}+\frac{\beta}{4} \cos \left(\xi / 2^{n}\right)
\end{array}\right) \\
\left(\begin{array}{c}
\hat{U}_{0}^{(n)}(\xi) \\
\hat{U}_{1}^{(n)}(\xi)
\end{array}\right)=i \frac{1-\beta}{2} \xi\left(\begin{array}{c}
\hat{T}_{0}^{(n-1)}(\xi) \\
\hat{T}_{1}^{(n-1)}(\xi)
\end{array}\right)+\frac{1+\beta}{2}\left(\begin{array}{c}
\hat{U}_{0}^{(n-1)}(\xi) \\
\hat{U}_{1}^{(n-1)}(\xi)
\end{array}\right)+O\left(1 / 2^{n}\right) \\
\left(\begin{array}{c}
\hat{U}_{0}^{(n)}(\xi) \\
\hat{U}_{1}^{(n)}(\xi)
\end{array}\right)=i \frac{1-\beta}{2} \xi \sum_{k=0}^{n-1}\left(\frac{1+\beta}{2}\right)^{k}\left(\begin{array}{c}
\hat{T}_{0}^{(n-1-k)}(\xi) \\
\hat{T}_{1}^{(n-1-k)}(\xi)
\end{array}\right)+O\left(n\left[\max \left(\frac{1}{2}, \frac{|1+\beta|}{2}\right)\right]^{n}\right)
\end{gathered}
$$

If $|1+\beta|<2$ which is the hypothesis, then the right member of the last vector equation tends to the column vector whose components are $i \xi \hat{T}_{0}(\xi), i \xi \hat{T}_{1}(\xi)$. They are the two respective limits of the sequences $\hat{U}_{0}^{(n)}(\xi), \hat{U}_{0}^{(n)}(\xi)$. Using the inverse Fourier transform on each sequence, it is clear that $U_{0}^{(n)}, U_{1}^{(n)}$ converge respectively to the distributions $T_{0}^{\prime}, T_{1}^{\prime}$. 
Theorem 11 Let us assume that $-3<\beta<1$. If we build the pair $(f, p)$ by the subdivision scheme (1) from the data $\left\{y_{k}, y_{k}^{\prime}\right\}_{k \in \mathbb{Z}}$, then the sequence of distributions $G_{n}=\frac{1}{2^{n}} \sum_{m=-\infty}^{\infty} p\left(m / 2^{n}\right) \delta_{m / 2^{n}}$ converges to the distribution

$$
G=\sum_{k=-\infty}^{\infty}\left[y_{k} \tau_{k} T_{0}^{\prime}+y_{k}^{\prime} \tau_{k} T_{1}^{\prime}\right]
$$

Proof: The proof is similar to that of Theorem 9.

We conclude this subsection by a comment of the referee. It is amazing that there is no restriction on the parameter $\alpha$ in Theorems 8-11. We think that this is typical of convergence in distribution and that for some values of $\alpha, C^{1}$-convergence will not happen.

\subsection{A characterization of Fourier transforms $\hat{T}_{0}, \hat{T}_{1}$}

In this subsection, we characterize the pair of functions $\hat{T}_{0}(\xi), \hat{T}_{1}(\xi)$ without using infinite iteration of the subdivision scheme for two systems of initial data.

Theorem 12 If $-5<\beta<3$, then $\left(\hat{T}_{0}(\xi), \hat{T}_{1}(\xi)\right.$ is the unique pair of analytic functions $\left(\phi_{0}(\xi), \phi_{1}(\xi)\right)$ which is a solution of the vector equation

$$
\left(\begin{array}{l}
\phi_{0}(2 \xi) \\
\phi_{1}(2 \xi)
\end{array}\right)=A(\xi)\left(\begin{array}{c}
\phi_{0}(\xi) \\
\phi_{1}(\xi)
\end{array}\right)
$$

and which satisfies $\phi_{0}(0)=1$.

Proof: Under the hypothesis $-5<\beta \leq 3$, we know that the sequence of matrices $P_{n}(\xi)$ converges. The limit $P$ satisfies the equation $P(2 \xi)=P(\xi) A(\xi)$ as it can be seen from Equation (5). By Theorem 8, $\left(\hat{T}_{0}(\xi), \hat{T}_{1}(\xi)\right)^{T}$ is the first column of the matrix $P(\xi)$, it follows that $\phi_{0}(\xi)=\hat{T}_{0}(\xi), \phi_{1}(\xi)=\hat{T}_{1}(\xi)$ provide a solution to the system of equations (10). Moreover we have $(\forall n \in \mathbb{N}) \hat{T}_{0}^{(n)}(0)=1$, hence $\phi_{0}(0)=1$.

Let $\left(\phi_{0}, \phi_{1}\right)$ be a pair of analytic functions solution of (10) and such that $\phi_{0}(0)=1$. By setting $\xi=0$ in equation (10), we have that $\phi_{1}(0)=0$ (because $\beta \neq 3$ ). We expand the functions $\phi_{0}(\xi), \phi_{1}(\xi)$ and the components $a_{i j}(\xi)$ of the matrix $A(\xi)$ in power series of $\xi$ :

$\phi_{0}(\xi)=\sum_{n=0}^{\infty} \phi_{0}^{(n)} \xi^{n}, \phi_{1}(\xi)=\sum_{n=0}^{\infty} \phi_{1}^{(n)} \xi^{n}, a_{i j}(\xi)=\sum_{n=0}^{\infty} a_{i j}^{(n)} \xi^{n}$.

Then we substitute these expansions in system (10). We develop the products and we reorganize the result in terms of powers of $\xi$. We use the hypothesis

$\phi_{0}(0)=1, \phi_{1}(0)=0$ to compute the Maclaurin series of the functions $\phi_{0}, \phi_{1}$ recursively, for $n=1,2,3, \ldots$ :

$$
\phi_{i-1}^{(n)}=\left(\sum_{j=1}^{2} \sum_{k=1}^{n} a_{i j}^{(k)} \phi_{j-1}^{(n-k)}\right) /\left(2^{n}-a_{i i}(0)\right), i=1,2
$$


Notice that $a_{11}(0)=1, a_{22}(0)=(1+\beta) / 4$. Therefore $\phi_{0}$ and $\phi_{1}$ are uniquely determined and are respectively $\hat{T}_{0}$ and $\hat{T}_{1}$.

Remark 2: In this last theorem, the analyticity hypothesis on the functions $\phi_{0}, \phi_{1}$ is critical. If one uses the continuity hypothesis combined with the values of $\phi_{0}(0), \phi_{1}(0)$ one does not have a unique solution of system (10). Kuczma proved in a general way in his book ([9], p. 245, Theorem 12.1), that the set of solutions of a system of type (10) depends on an arbitrary function.

\section{The Hermite subdivision scheme $H S 22$}

We will see how we can generalize the previous results to the scheme $H S 22$ which is based on bisections that use not only the values of a function and of its first derivative but also the values of its second derivative. We recall Merrien's construction [11]. If the values of a function $f$, and of its first and second derivative $p, s$ are known on $\mathbb{Z}$, we build the functions $f, p$ and $s$ by a recursion on $n$ as in the previous section. If $a=j / 2^{n}$ and $b=(j+1) / 2^{n}$ are two successive points of $D_{n}$, we compute $f, p$ and $s$ at the midpoint $x=(a+b) / 2$ by the formulae:

$$
\left.\begin{array}{l}
f(x)=1 / 2[f(b)+f(a)]+\alpha_{2} h[p(b)-p(a)]+\alpha_{3} h^{2}[s(b)+s(a)] \\
p(x)=\beta_{1} \frac{f(b)-f(a)}{h}+\beta_{2}[p(a)+p(b)]+\beta_{3} h[s(b)-s(a)] \\
s(x)=\gamma_{2} \frac{p(b)-p(a)}{h}+\gamma_{3}[s(b)+s(a)]
\end{array}\right\}
$$

The algorithm is said to be $C^{2}$-convergent if, for any data $\left\{y_{k}, y_{k}^{\prime}, y_{k}^{\prime \prime}\right\}_{k \in \mathbb{Z}}$, the three functions $f, p, s$ can be extended from $D_{\infty}$ to $\mathbb{R}$ with $f \in C^{2}(\mathbb{R}), p=f^{\prime}$ and $s=f^{\prime \prime}$. The second author found a necessary condition to get $C^{2}$-convergence (Proposition 3 in $[11])$ :

$$
8 \alpha_{2}+16 \alpha_{3}=-1, \beta_{1}+2 \beta_{2}=1, \gamma_{2}+2 \gamma_{3}=1 .
$$

We will find again the last two conditions later in the convergence theorems in the space of distributions. If these conditions are satisfied, then the algorithm depends only on 4 parameters: $\alpha_{2}, \beta_{1}, \beta_{3}$ and $\gamma_{2}$.

As for $H S 21$, with this necessary hypothesis, a necessary and sufficient condition to get $C^{2}$-convergence is $\hat{\rho}(\Sigma)<1$ where $\Sigma=\left\{\Lambda_{1}, \Lambda_{-1}\right\}$ and

$$
\Lambda_{\varepsilon}=\left(\begin{array}{ccc}
\frac{1}{2} & \varepsilon \gamma_{2} & 0 \\
\varepsilon\left(\frac{1}{4}+2 \beta_{3}-\frac{\beta_{1}}{6}\right) & 1-\frac{\gamma_{2}}{2} & \varepsilon 2 \beta_{1} \\
-\frac{1}{8}+\beta_{3}-\frac{\beta_{1}}{12} & \varepsilon\left(\frac{1}{2}+4 \alpha_{2}+\frac{\gamma_{2}}{12}\right) & 2-\beta_{1}
\end{array}\right), \varepsilon \pm 1 .
$$

An equivalent condition is that there exists a matrix norm $\|\cdot\|$ such that: $\left\|\Lambda_{\varepsilon}\right\|<1, \varepsilon= \pm 1$. The proof of this result is given in [11].

Remark 3: For some values of the parameters, the function $f$ on each interval $[k, k+1], k \in \mathbb{Z}$ is a usual polynomial or a piecewise polynomial. For example: 
1. $\alpha_{2}=-5 / 32, \alpha_{3}=1 / 64, \beta_{1}=15 / 8, \beta_{2}=-7 / 16, \beta_{3}=1 / 32, \gamma_{2}=3 / 2$, $\gamma_{3}=-1 / 4$, then $f$ is the Hermite interpolating quintic polynomial on each interval $[k, k+1], k \in \mathbb{Z}$,

2. $\alpha_{2}=-23 / 144, \alpha_{3}=5 / 288, \beta_{1}=9 / 4, \beta_{2}=-5 / 8, \beta_{3}=1 / 16, \gamma_{2}=3 / 2$, $\gamma_{3}=-1 / 4$, then $f$ is the cubic spline with knots at $\mathbb{Z} / 3$,

3. $\alpha_{2}=-5 / 32, \alpha_{3}=1 / 64, \beta_{1}=2, \beta_{2}=-1 / 2, \beta_{3}=1 / 24, \gamma_{2}=3 / 2, \gamma_{3}=-1 / 4$, then $f$ is the quartic spline with knots at $\mathbb{Z} / 2$.

\subsection{Elementary properties of $H S 22$}

Of course, we have lemmas equivalent to Lemma 1 and 2 on linearity, translation and scale change for the scheme $H S 22$; they are not written again.

As for $H S 21$, we introduce three basic solutions for the recursive system (12): the triples $f_{i}, p_{i}, s_{i}, i=0,1,2$ which are solutions of (12) with data,

$$
\forall k \in \mathbb{Z}, \begin{aligned}
& f_{0}(k)=\delta_{k, 0}, p_{0}(k)=0, s_{0}(k)=0 \\
& f_{1}(k)=0, p_{1}(k)=\delta_{k, 0}, s_{1}(k)=0 \\
& f_{2}(k)=0, p_{2}(k)=0, s_{2}(k)=\delta_{k, 0}
\end{aligned}
$$

Then, for any construction produced by (12), we have for any dyadic number $x$ :

$$
\left.\begin{array}{l}
f(x)=\sum_{k=-\infty}^{\infty}\left[f(k) f_{0}(x-k)+p(k) f_{1}(x-k)+s(k) f_{2}(x-k)\right] \\
p(x)=\sum_{k=-\infty}^{\infty}\left[f(k) p_{0}(x-k)+p(k) p_{1}(x-k)+s(k) p_{2}(x-k)\right] \\
s(x)=\sum_{k=-\infty}^{\infty}\left[f(k) s_{0}(x-k)+p(k) s_{1}(x-k)+s(k) s_{2}(x-k)\right]
\end{array}\right\}
$$

From these formulae we can deduce a system of functional equations:

$$
\begin{aligned}
f_{0}(x / 2)= & f_{0}(x-1) / 2+f_{0}(x)+f_{0}(x+1) / 2+\frac{\beta_{1}}{2}\left[-f_{1}(x-1)+f_{1}(x+1)\right] \\
f_{1}(x / 2)= & \alpha_{2}\left[-f_{0}(x-1)+f_{0}(x+1)\right]+\frac{\beta_{2}}{2} f_{1}(x-1)+\frac{1}{2} f_{1}(x)+\frac{\beta_{2}}{2} f_{1}(x+1) \\
& +\frac{\gamma_{2}}{4}\left[-f_{2}(x-1)+f_{2}(x+1)\right] \\
f_{2}(x / 2)= & \alpha_{3}\left[f_{0}(x-1)+f_{0}(x+1)\right]+\frac{\beta_{3}}{2}\left[-f_{1}(x-1)+f_{1}(x+1)\right] \\
& \left.+\frac{\gamma_{3}}{4} f_{2}(x-1)+\frac{1}{4} f_{2}(x)+\frac{\gamma_{3}}{4} f_{2}(x+1)\right]
\end{aligned}
$$

and similar equations in terms of $p_{i}$ and $s_{i}(\operatorname{cf}(3))$. 


\subsection{Fourier transform of $H S 22$}

When the Fourier operator is applied to each equation of the system (14) and to similar equations for $p_{i}, s_{i}$, we obtain

$$
\begin{gathered}
\left(\begin{array}{c}
\hat{f}_{0}(\xi) \\
\hat{f}_{1}(\xi) \\
\hat{f}_{2}(\xi)
\end{array}\right)=A(\xi / 2)\left(\begin{array}{c}
\hat{f}_{0}(\xi / 2) \\
\hat{f}_{1}(\xi / 2) \\
\hat{f}_{2}(\xi / 2)
\end{array}\right),\left(\begin{array}{c}
\hat{p}_{0}(\xi) \\
\hat{p}_{1}(\xi) \\
\hat{p}_{2}(\xi)
\end{array}\right)=2 A(\xi / 2)\left(\begin{array}{c}
\hat{p}_{0}(\xi / 2) \\
\hat{p}_{1}(\xi / 2) \\
\hat{p}_{2}(\xi / 2)
\end{array}\right), \\
\left(\begin{array}{c}
\hat{s}_{0}(\xi) \\
\hat{s}_{1}(\xi) \\
\hat{s}_{2}(\xi)
\end{array}\right)=4 A(\xi / 2)\left(\begin{array}{c}
\hat{s}_{0}(\xi / 2) \\
\hat{s}_{1}(\xi / 2) \\
\hat{s}_{2}(\xi / 2)
\end{array}\right),
\end{gathered}
$$

where

$$
A(\xi)=\left(\begin{array}{ccc}
\frac{1}{2}+\frac{1}{2} \cos \xi & i \frac{\beta_{1}}{2} \sin \xi & 0 \\
i \alpha_{2} \sin \xi & \frac{1}{4}+\frac{\beta_{2}}{2} \cos \xi & i \frac{\gamma_{2}}{4} \sin \xi \\
\alpha_{3} \cos \xi & i \frac{\beta_{3}}{2} \sin \xi & \frac{1}{8}+\frac{\gamma_{3}}{4} \cos \xi
\end{array}\right)
$$

Therefore, again, we will have to study the sequence of matrix products:

$$
P_{n}(\xi)=A(\xi / 2) A(\xi / 4) \ldots A\left(\xi / 2^{n}\right) .
$$

Using Lemma 4 and Theorem 5, we prove a similar theorem to Theorem 6 and its corollary. But before that, we must change the matrix norm.

Let $\Theta=\left(\theta_{1}, \theta_{2}, \ldots, \theta_{d}\right)$ be an element of $\mathbb{R}^{d}$ with positive $\theta_{i}$. We define a new norm $\|\cdot\|_{\Theta}$ on $\mathbb{C}^{d}$ by $\|X\|_{\Theta}=\max _{i=1, d}\left(\theta_{i}\left|x_{i}\right|\right), X \in \mathbb{C}^{d}$. It is easy to prove that the associated matrix norm satisfies $\|A\|_{\Theta}=\max _{i} \sum_{j} \theta_{i} / \theta_{j}\left|a_{i j}\right|$ whenever $A=\left(a_{i j}\right)$ lies in $\mathbb{C}^{d \times d}$. Obviously if $\theta_{1}=\ldots=\theta_{d}=1$, we obtain the usual norm \|\|$_{\infty}$.

Theorem 13 If $-5 / 2<\beta_{2} \leq 3 / 2$ and if $-9 / 2<\gamma_{3}<7 / 2$, then for any complex number $\xi$, the sequence of matrices $P_{n}(\xi)$ defined in (16) converges and the convergence is uniform on any boounded domain. The nine components of the limit matrix $P(\xi)$ are entire functions of exponential type $\leq 1$.

Proof: We proceed as in Theorem 6 except that on $\mathbb{C}^{3}$, we use the norm $\|X\|_{\Theta}$ with $\Theta=\left(1,1, \theta_{3}\right)$ where $\theta_{3}$ is chosen small enough to get $\theta_{3}\left|\alpha_{3}\right|+\left|1 / 8+\gamma_{3} / 4\right| \leq 1$. Then it is easy to prove that $\left\|A\left(z / 2^{n}\right)\right\|_{\Theta} \leq 1+O\left(1 / 2^{n}\right)$ in the disk $|z| \leq R$.

Notice that $A(0)=\left(\begin{array}{ccc}1 & 0 & 0 \\ 0 & \frac{1}{4}+\frac{\beta_{2}}{2} & 0 \\ \alpha_{3} & 0 & \frac{1}{8}+\frac{\gamma_{3}}{4}\end{array}\right)$ so that $\|A(0)\|_{\Theta}=1$ with the above condition on $\theta_{3}$.

By induction, we prove that

$$
[A(0)]^{n}=\left(\begin{array}{ccc}
1 & 0 & 0 \\
0 & \left(\frac{1}{4}+\frac{\beta_{2}}{2}\right)^{n} & 0 \\
\frac{\alpha_{3}}{\frac{1}{8}+\frac{\gamma_{3}}{4}-1}\left[\left(\frac{1}{8}+\frac{\gamma_{3}}{4}\right)^{n}-1\right] & 0 & \left(\frac{1}{8}+\frac{\gamma_{3}}{4}\right)^{n}
\end{array}\right), n \geq 1
$$


therefore, using the hypothesis, this sequence converges to the matrix $\left(\begin{array}{rrr}1 & 0 & 0 \\ 0 & 0 & 0 \\ -\frac{\alpha_{3}}{\frac{1}{8}+\frac{\gamma_{3}}{4}-1} & 0 & 0\end{array}\right)$.

We can complete the proof as in the previous section because the hypotheses of Theorem 5 are satisfied.

Corollary 14 If $-5 / 2<\beta_{2} \leq 3 / 2$ and if $-9 / 2<\gamma_{3}<7 / 2$, then each component of the limit matrix $P(z)=\lim P_{n}(z)$ is the Fourier transform of a distribution with support in the interval $[-1,1]$.

\subsection{Schwartz distributions associated to $H S 22$}

We introduce 9 sequences of distributions in Schwartz sense:

$$
\begin{aligned}
T_{i}^{(n)} & =\frac{1}{2^{n}} \sum_{m} f_{i}\left(m / 2^{n}\right) \delta_{m / 2^{n}}, i=0,1,2, \\
U_{i}^{(n)} & =\frac{1}{2^{n}} \sum_{m} p_{i}\left(m / 2^{n}\right) \delta_{m / 2^{n}}, i=0,1,2, \\
V_{i}^{(n)} & =\frac{1}{2^{n}} \sum_{m} s_{i}\left(m / 2^{n}\right) \delta_{m / 2^{n}}, i=0,1,2 .
\end{aligned}
$$

When we compute the Fourier transform of these distributions, we obtain:

$$
\hat{T}_{i}^{(n)}(\xi)=T_{i}^{(n)}\left(e^{-i \xi x}\right), \hat{U}_{i}^{(n)}(\xi)=U_{i}^{(n)}\left(e^{-i \xi x}\right), \hat{V}_{i}^{(n)}(\xi)=V_{i}^{(n)}\left(e^{-i \xi x}\right) .
$$

Then using the equations (14), we may verify that three recursions link these Fourier transform through the matrix $A(\xi)$ :

$$
\begin{gathered}
\left(\begin{array}{c}
\hat{T}_{0}^{(n+1)}(\xi) \\
\hat{T}_{1}^{(n+1)}(\xi) \\
\hat{T}_{2}^{(n+1)}(\xi)
\end{array}\right)=A(\xi / 2)\left(\begin{array}{c}
\hat{T}_{0}^{(n)}(\xi / 2) \\
\hat{T}_{1}^{(n)}(\xi / 2) \\
\hat{T}_{2}^{(n)}(\xi / 2)
\end{array}\right),\left(\begin{array}{c}
\hat{U}_{0}^{(n+1)}(\xi) \\
\hat{U}_{1}^{(n+1)}(\xi) \\
\hat{U}_{2}^{(n+1)}(\xi)
\end{array}\right)=2 A(\xi / 2)\left(\begin{array}{c}
\hat{U}_{0}^{(n)}(\xi / 2) \\
\hat{U}_{1}^{(n)}(\xi / 2) \\
\hat{U}_{2}^{(n)}(\xi / 2)
\end{array}\right) \\
\left(\begin{array}{c}
\hat{V}_{0}^{(n+1)}(\xi) \\
\hat{V}_{1}^{(n+1)}(\xi) \\
\hat{V}_{2}^{(n+1)}(\xi)
\end{array}\right)=4 A(\xi / 2)\left(\begin{array}{c}
\hat{V}_{0}^{(n)}(\xi / 2) \\
\hat{V}_{1}^{(n)}(\xi / 2) \\
\hat{V}_{2}^{(n)}(\xi / 2)
\end{array}\right) \\
\operatorname{As}\left(\begin{array}{c}
1 \\
\hat{T}_{0}^{(0)}(\xi) \\
\hat{T}_{1}^{(0)}(\xi) \\
\hat{T}_{2}^{(0)}(\xi)
\end{array}\right)=\left(\begin{array}{c}
1 \\
0 \\
0
\end{array}\right),\left(\begin{array}{c}
\hat{U}_{0}^{(0)}(\xi) \\
\hat{U}_{1}^{(0)}(\xi) \\
\hat{U}_{2}^{(0)}(\xi)
\end{array}\right)=\left(\begin{array}{c}
0 \\
1 \\
0
\end{array}\right),\left(\begin{array}{c}
\hat{V}_{0}^{(0)}(\xi) \\
\hat{V}_{1}^{(0)}(\xi) \\
\hat{V}_{2}^{(0)}(\xi)
\end{array}\right)=\left(\begin{array}{c}
0 \\
0 \\
1
\end{array}\right), \text { we de- }
\end{gathered}
$$

duce that

$$
\left(\begin{array}{c}
\hat{T}_{0}^{(n)}(\xi) \\
\hat{T}_{1}^{(n)}(\xi) \\
\hat{T}_{2}^{(n)}(\xi)
\end{array}\right)=P_{n}(\xi)\left(\begin{array}{l}
1 \\
0 \\
0
\end{array}\right)
$$

and 


$$
\left(\begin{array}{c}
\hat{U}_{0}^{(n)}(\xi) \\
\hat{U}_{1}^{(n)}(\xi) \\
\hat{U}_{2}^{(n)}(\xi)
\end{array}\right)=2^{n} P_{n}(\xi)\left(\begin{array}{c}
0 \\
1 \\
0
\end{array}\right),\left(\begin{array}{c}
\hat{V}_{0}^{(n)}(\xi) \\
\hat{V}_{1}^{(n)}(\xi) \\
\hat{V}_{2}^{(n)}(\xi)
\end{array}\right)=4^{n} P_{n}(\xi)\left(\begin{array}{c}
0 \\
0 \\
1
\end{array}\right) .
$$

Like Theorems 8 and 9 for $H S 21$, we have the two following theorems whose proofs are similar.

Theorem 15 If $-5 / 2<\beta_{2} \leq 3 / 2$ and if $-9 / 2<\gamma_{3}<7 / 2$, then the three sequences of distributions $T_{0}^{(n)}, T_{1}^{(n)}, T_{2}^{(n)}$ converge respectively to the distributions $T_{0}, T_{1}, T_{2}$. The vector $\left(\hat{T}_{0}(\xi), \hat{T}_{1}(\xi), \hat{T}_{2}(\xi)\right)^{T}$ is the first column of the matrix $P(\xi)$.

Theorem 16 We suppose that $-5 / 2<\beta_{2} \leq 3 / 2$ et $-9 / 2<\gamma_{3}<7 / 2$. If we build the triple $(f, p, s)$ by the subdivision scheme (12) from the data $\left\{y_{k}, y_{k}^{\prime}, y_{k}^{\prime \prime}\right\}_{k \in \mathbb{Z}}$, then the sequence of distributions $F_{n}=\frac{1}{2^{n}} \sum_{m=-\infty}^{\infty} f\left(m / 2^{n}\right) \delta_{m / 2^{n}}$ converges to the distribution

$$
F=\sum_{k=-\infty}^{\infty}\left[y_{k} \tau_{k} T_{0}+y_{k}^{\prime} \tau_{k} T_{1}+y_{k}^{\prime \prime} \tau_{k} T_{2}\right]
$$

To study the convergence of the sequences $\hat{U}_{i}^{(n)}$ and $\hat{V}_{i}^{(n)}, i=0,1,2$, we need a new lemma which gives the convergence of an infinite product of matrices.

Lemma 17 Let $M=\left(\begin{array}{lll}1 & 0 & 0 \\ a & b & 0 \\ 0 & c & d\end{array}\right) \in \mathbb{C}^{3 \times 3}$ with $|b|<1,|d|<1$ then

$$
\lim _{n \rightarrow+\infty} M^{n}=\left(\begin{array}{ccc}
1 & 0 & 0 \\
\frac{a}{1-b} & 0 & 0 \\
\frac{a c}{(1-b)(1-d)} & 0 & 0
\end{array}\right)
$$

Proof: By induction, we obtain that for all $n \geq 2$ and $b \neq d$

$$
M^{n}=\left(\begin{array}{ccc}
1 & 0 & 0 \\
a \frac{1-b^{n}}{1-b} & b^{n} & 0 \\
\frac{a c}{b-d}\left(\frac{1-b^{n}}{1-b}-\frac{1-d^{n}}{1-d}\right) & c \frac{b^{n}-d^{n}}{b-d} & d^{n}
\end{array}\right)
$$

and for $b=d$,

$$
M^{n}=\left(\begin{array}{ccc}
1 & 0 & 0 \\
a \frac{1-b^{n}}{1-b} & b^{n} & 0 \\
a c \frac{(n-1) b^{n}-n b^{n-1}+1}{(1-b)^{2}} & c n b^{n-1} & b^{n}
\end{array}\right)
$$

It is now easy to conclude. 
Theorem 18 If $-3 / 2<\beta_{2}<1 / 2$ and $-3 / 2<\gamma_{3}<1 / 2$ then both sequences $U_{i}^{(n)}$ and $V_{i}^{(n)}, i=0,1,2$ converge respectively to $U_{i}$ and $V_{i}$.

If we add $\beta_{1}+2 \beta_{2}=1$, then $U_{i}=T_{i}^{\prime}, i=0,1,2$

Moreover, if we add $\gamma_{2}+2 \gamma_{3}=1$, then $V_{i}=T_{i}^{\prime \prime}, i=0,1,2$.

Proof: Let us set $t_{n}=\left(\begin{array}{c}\hat{T}_{0}^{(n)} \\ \hat{T}_{1}^{(n)} \\ \hat{T}_{2}^{(n)}\end{array}\right), u_{n}=\left(\begin{array}{c}\hat{U}_{0}^{(n)} \\ \hat{U}_{1}^{(n)} \\ \hat{U}_{2}^{(n)}\end{array}\right), v_{n}=\left(\begin{array}{c}\hat{V}_{0}^{(n)} \\ \hat{V}_{1}^{(n)} \\ \hat{V}_{2}^{(n)}\end{array}\right)$. With (17), we get

$$
\begin{aligned}
t_{n} & =P_{n-1} A\left(\xi / 2^{n}\right)\left(\begin{array}{l}
1 \\
0 \\
0
\end{array}\right)=P_{n-1}\left(\begin{array}{c}
1 / 2+\cos \left(\xi / 2^{n}\right) / 2 \\
i \alpha_{2} \sin \left(\xi / 2^{n}\right) \\
\alpha_{3} \cos \left(\xi / 2^{n}\right)
\end{array}\right) \\
& =\left[1 / 2+\cos \left(\xi / 2^{n}\right) / 2\right] t_{n-1}+\frac{1}{2^{n-1}} i \alpha_{2} \sin \left(\xi / 2^{n}\right) u_{n-1}+\frac{1}{4^{n-1}} \alpha_{3} \cos \left(\xi / 2^{n}\right) v_{n-1} .
\end{aligned}
$$

Similarly, we obtain:

$$
\begin{aligned}
& u_{n}=i \frac{\beta_{1}}{2} 2^{n} \sin \left(\xi / 2^{n}\right) t_{n-1}+\left[1 / 2+\beta_{2} \cos \left(\xi / 2^{n}\right)\right] u_{n-1}+i \frac{\beta_{3}}{2} 2^{n} \sin \left(\xi / 2^{n}\right) \frac{1}{4^{n-1}} v_{n-1} \\
& v_{n}=i \frac{\gamma_{2}}{2} 2^{n} \sin \left(\xi / 2^{n}\right) u_{n-1}+\left[1 / 2+\gamma_{3} \cos \left(\xi / 2^{n}\right)\right] v_{n-1} .
\end{aligned}
$$

These equations can be written in a vector form: $\left(\begin{array}{c}t_{n} \\ u_{n} \\ v_{n}\end{array}\right)=N_{n}\left(\begin{array}{c}t_{n-1} \\ u_{n-1} \\ v_{n-1}\end{array}\right)$ where

$$
N_{n}=\left(\begin{array}{ccc}
1 / 2+\cos \left(\xi / 2^{n}\right) / 2 & \frac{1}{2^{n-1}} i \alpha_{2} \sin \left(\xi / 2^{n}\right) & \frac{1}{4^{n-1}} i \alpha_{3} \cos \left(\xi / 2^{n}\right) \\
i \frac{\beta_{1}}{2} 2^{n} \sin \left(\xi / 2^{n}\right) & 1 / 2+\beta_{2} \cos \left(\xi / 2^{n}\right) & i \beta_{3} \frac{1}{2^{n-1}} \sin \left(\xi / 2^{n}\right) \\
0 & i \frac{\gamma_{2}}{2} 2^{n} \sin \left(\xi / 2^{n}\right) & 1 / 2+\gamma_{3} \cos \left(\xi / 2^{n}\right)
\end{array}\right)
$$

Setting $M=\left(\begin{array}{ccc}1 & 0 & 0 \\ i \frac{\beta_{1}}{2} \xi & 1 / 2+\beta_{2} & 0 \\ 0 & i \frac{\gamma_{2}}{2} \xi & 1 / 2+\gamma_{3}\end{array}\right)$ and using the previous lemma and the hypothesis on $\beta_{2}$ and $\gamma_{3}$, we get that the sequence $M^{n}$ converges.

We choose a matrix norm $\|\cdot\|_{\Theta}=\left(1, \theta_{2}, \theta_{3}\right)$ with $\theta_{2}$ and $\theta_{3}$ such that:

$\theta_{2}\left|\frac{\beta_{1}}{2} \xi\right|+\left|1 / 2+\beta_{2}\right|<1$ then $\frac{\theta_{3}}{\theta_{2}}\left|\frac{\gamma_{2}}{2} \xi\right|+\left|1 / 2+\gamma_{3}\right|<1$

Now $\|M\|_{\Theta}=1$ and $\left\|M-N_{n}\right\|_{\Theta}=O\left(1 / 2^{n}\right)$. Using again Theorem 5 we conclude that the sequence $N_{n} N_{n-1} \ldots N_{1}$ converges, so that the sequence $\left(t_{n}, u_{n}, v_{n}\right)^{T}$ converges to $(t, u, v)^{T}$.

When we reach the limit, then $u=i \frac{\beta_{1}}{2} \xi t+\left(1 / 2+\beta_{2}\right) u$ and $v=i \frac{\gamma_{2}}{2} \xi u+\left(1 / 2+\gamma_{3}\right) v$. This can be written $u=i \frac{\beta_{1}}{1-2 \beta_{2}} \xi t$ and $v=i \frac{\gamma_{2}}{1-2 \gamma_{3}} \xi u$. By the inverse Fourier transform, we have the convergence result for $U_{i}^{(n)}$ and $V_{i}^{(n)}, i=0,1,2$. 
With the additional hypothesis $\beta_{1}+2 \beta_{2}=1$, we have $u=i \xi t$, therefore $U_{i}=T_{i}^{\prime}, i=0,1,2$.

Finally, if moreover $\gamma_{2}+2 \gamma_{3}=1$, then $v=i \xi u$ so that $V_{i}=T_{i}^{\prime \prime}, i=0,1,2$.

Now we have a last theorem on the convergence of derivatives of distributions as we had for $H S 21$.

Theorem 19 We suppose that $-3 / 2<\beta_{2}<1 / 2,-3 / 2<\gamma_{3}<1 / 2$ and $\beta_{1}+2 \beta_{2}=$ $1, \gamma_{2}+2 \gamma_{3}=1$. If we build the triple $(f, p, s)$ by the subdivision scheme (12) from the data $\left\{y_{k}, y_{k}^{\prime}, y_{k}^{\prime \prime}\right\}_{k \in \mathbb{Z}}$, then the sequence of distributions $G_{n}=\frac{1}{2^{n}} \sum_{m=-\infty}^{\infty} p\left(m / 2^{n}\right) \delta_{m / 2^{n}}$ converges to the distribution $G=\sum_{k=-\infty}^{\infty}\left[y_{k} \tau_{k} T_{0}^{\prime}+y_{k}^{\prime} \tau_{k} T_{1}^{\prime}+y_{k}^{\prime \prime} \tau_{k} T_{2}^{\prime}\right]$,

and the sequence of distributions $H_{n}=\frac{1}{2^{n}} \sum_{m=-\infty}^{\infty} s\left(m / 2^{n}\right) \delta_{m / 2^{n}}$ converges to the distribution $H=\sum_{k=-\infty}^{\infty}\left[y_{k} \tau_{k} T_{0}^{\prime \prime}+y_{k}^{\prime} \tau_{k} T_{1}^{\prime \prime}+y_{k}^{\prime \prime} \tau_{k} T_{2}^{\prime \prime}\right]$.

\subsection{A characterization of Fourier transforms $\hat{T}_{0}, \hat{T}_{1}, \hat{T}_{2}$}

We characterize the triple of functions $\phi_{i}(\xi)=\hat{T}_{i}(\xi), i=0,1,2$ without computing all the subdivision scheme for three triples of initial data.

Theorem 20 If $-5 / 2<\beta_{2}<3 / 2$ and $-9 / 2<\gamma_{3}<7 / 2$, then $\left(\hat{T}_{0}(\xi), \hat{T}_{1}(\xi), \hat{T}_{2}(\xi)\right)$ is the unique triple of analytic functions $\left(\phi_{0}(\xi), \phi_{1}\left(\xi, \phi_{2}(\xi)\right)\right)$ which is a solution of the vector equation

$$
\left(\begin{array}{c}
\phi_{0}(2 \xi) \\
\phi_{1}(2 \xi) \\
\phi_{2}(2 \xi)
\end{array}\right)=A(\xi)\left(\begin{array}{c}
\phi_{0}(\xi) \\
\phi_{1}(\xi) \\
\phi_{2}(\xi)
\end{array}\right)
$$

and which satisfies $\phi_{0}(0)=1$.

Proof: One must first notice that the matrix $A(0)$ is a lower triangular matrix whose diagonal components are successively $1,1 / 4+\beta / 2,1 / 8+\gamma_{3} / 4$. The proof that $\left.\left(\phi_{0}=\hat{T}_{0}, \phi_{1}=\hat{T}_{1}, \phi_{2} \hat{T}_{2}\right)\right)$ which is a solution of the vector equation (19) is entirely similar to that one of Theorem 12. By setting $\xi=0$ in Equation (19),we also remark that $\left(\hat{T}_{0}(0), \hat{T}_{1}(0) \hat{T}_{1}(0)\right)^{T}$ is a column eigenvector for the eigenvalue 1 of the matrix $\mathrm{A}(0)$.

Now, let $\left(\phi_{0}, \phi_{1}, \phi_{2}\right)$ be a triple of analytic functions solution of (19) and such that $\phi_{0}(0)=1$. As in Theorem 12, we expand the functions $\phi_{0}, \phi_{1}, \phi_{2}$ and the components of $A(\xi)$ in power series of $\xi$ :

$\phi_{i}(\xi)=\sum_{n=0}^{\infty} \phi_{i}^{(n)} \xi^{n}, i=1,2,3 ; A(\xi)=\sum_{n=0}^{\infty} \xi^{n} A_{n}$. 
When we substitute 0 to $\xi$ in equation (19), we obtain that $\left(\phi_{0}(0), \phi_{1}(0), \phi_{2}(0)\right)^{T}$ is a column eigenvector for the eigenvalue 1 of the matrix $A(0)$. Since $A(0)$ is triangular with only one 1 on its main diagonal, this eigenvector is unique when its first component is 1 . Hence $\phi_{i}(0)=\hat{T}_{i}(0), i=0,1,2$. By replacing $\phi_{i}$ with their powers series in the system (19), by developing the products and reorganizing the results in terms of powers of $\xi$, we obtain the series of equations depending on $n=1,2,3, \ldots$ :

$$
2^{n}\left(\begin{array}{c}
\phi_{0}^{(n)} \\
\phi_{1}^{(n)} \\
\phi_{2}^{(n)}
\end{array}\right)=\sum_{k=0}^{n} A_{k}\left(\begin{array}{c}
\phi_{0}^{(n-k)} \\
\phi_{1}^{(n-k)} \\
\phi_{2}^{(n-k)}
\end{array}\right),
$$

Since for all $n>0$, the matrix $2^{n} I-A(0)$ has an inverse, all the components in the expansions of $\phi_{i}^{(n)}, i=0,1,2$ are uniquely determined. Hence $\phi_{i}=\hat{T}_{i}, i=0,1,2$.

\section{Conclusion}

We defined the notion of convergence in distribution of Hermite subdivision schemes and we studied two classes of subdivision schemes for their convergence. For each class, we were able to find a large region of the parameter space for which distributional convergence happens. Is it possible to enlarge the region for which convergence in distribution will be verified? More generally, is it possible to find simple necessary and sufficient conditions for distributional convergence for any Hermite subdivision scheme?

After defining Hermite subdivision schemes on the space of distributions, one may think of many other questions. One of these is the following. In the scheme $H S 11$, can one characterize the largest region of the parameters $\alpha, \beta$ such that for

the corresponding Fourier transforms $\hat{T}_{0}, \hat{T}_{1}, \int_{-\infty}^{\infty}\left(1+\xi^{2}\right)\left|\hat{T}_{i}(\xi)\right|^{2} d \xi<\infty, i=0,1$ ? This characterization would be useful to specifying all interpolating functions $f$ of the scheme such that $f$ and $f^{\prime}$ are in $L^{2}(\mathbb{R})$.

\section{References}

[1] M. Artzrouni, On the convergence of infinite products of matrices. Linear Algebra Appl. 74 (1986) 11-21.

[2] I. Daubechies and J. C. Lagarias, Set of matrices all infinite products of which converge. Linear Algebra Appl. 161 (1992) 227-263.

[3] G. Deslauriers, S. Dubuc, Transformées de Fourier de courbes irrégulières. Ann. Sc. Math. Québec 11 (1987) 25-44. 
[4] G. Deslauriers, J. Dubois, S. Dubuc, Multidimensional Iterative Interpolation. Canad. J. Math. 43 (1991) 297-312.

[5] N. Dyn, D. Levin, Analysis of Hermite-type Subdivision Schemes. In Approximation Theory VIII. Vol 2: Wavelets and Multilevel Approximation, Ed: C. K. Chui and L.L. Schumaker. World Scientific, Singapore, 1995, pp. 117-124.

[6] N. Dyn, D. Levin, Analysis of Hermite-interpolatory subdivision schemes. In Spline Functions and the Theory of Wavelets, S. Dubuc and G. Deslauriers, Ed. Amer. Math. Soc., Providence R. I., 1999, pp. 105-113.

[7] L. Hervé, Multi-Resolution Analysis of Multiplicity d: Applications to Dyadic Interpolation. Applied Comput. Harmon. Anal. 1 (1994) 299-315.

[8] L. Kobbelt, Using the discete Fourier transform to analyse the convergence of subdivision schemes. Applied Comput. Harmon. Anal. 5 (1998) 68-91.

[9] M. Kuczma, Functional Equations in a Single Variable. Państwowe Wydawnictwo Naukowe, Warsaw, 1968.

[10] J.-L. Merrien, A family of Hermite interpolants by bissection algorithms. Numer. Algorithms 2 (1992) 187-200.

[11] J.-L. Merrien, Interpolants d'Hermite $C^{2}$ obtenus par subdivision. M2AN Math. Model. Numer. Anal. 33 (1999) 55-65.

[12] L. Schwartz, Théorie des distributions, Tomes 1 and 2. Hermann, Paris, 1956. 